\title{
Magnetic field structure of large-scale plasmoid generated by the fast reconnection mechanism in a sheared current sheet
}

\author{
M. Ugai \\ Research Center for Space and Cosmic Evolution, Ehime University, Matsuyama 790-8577, Japan \\ Received: 24 June 2010 - Revised: 16 July 2010 - Accepted: 2 August 2010 - Published: 11 August 2010
}

\begin{abstract}
On the basis of the spontaneous fast reconnection model, three-dimensional magnetic field profiles associated with a large-scale plasmoid propagating along the antiparallel magnetic fields are studied in the general sheared current sheet system. The plasmoid is generated ahead of the fast reconnection jet as a result of distinct compression of the magnetized plasma. Inside the plasmoid, the sheared (east-west) field component has the peak value at the plasmoid center located at $x=X_{\mathrm{C}}$, where the north-south field component changes its sign. The plasmoid center corresponds to the socalled contact discontinuity that bounds the reconnected field lines in $x<X_{\mathrm{C}}$ and the field lines without reconnection in $x>X_{\mathrm{C}}$. Hence, contray to the conventional prediction, the reconnected sheared field lines in $x<X_{\mathrm{C}}$ are not spiral or helical, since they cannot be topologically connected to the field lines in $x>X_{\mathrm{C}}$. It is demonstrated that the resulting profiles of magnetic field components inside the plasmoid are, in principle, consistent with satellite observations. In the ambient magnetic field region outside the plasmoid too, the magnetic field profiles are in good agreement with the well-known observations of traveling compression regions (TCRs).
\end{abstract}

Keywords. Magnetospheric physics (Storms and substorms)

\section{Introduction}

Magnetic reconnection is essential for large dissipative events in space plasmas, such as solar flares and geomagnetic substorms (Klimchuk, 2006; Nakamura et al., 2006; Sharma et al., 2008). In view of the explosive magnetic energy conversion involved, the fast reconnection mechanism,

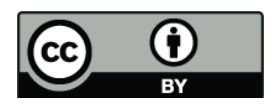

Correspondence to: M. Ugai

(ugai@cosmos.ehime-u.ac.jp) involving Alfvénic plasma jets by standing slow shocks, is most responsible for these flare phenomena (Shibata, 1999; Uzdensky, 2003; Ugai, 2008b). Historically, possible fast reconnection configurations were proposed on the basis of two-dimensional (2-D) steady magnetohydrodynamic (MHD) equations, and it was argued that the reconnection rate should be controlled by external boundary conditions (Petschek, 1963; Vasyliunas, 1975; Priest and Forbes, 1986). On the other hand, Ugai and Tsuda (1977) demonstrated for the first time that the fast reconnection mechanism can be realized in a 2-D current sheet system as an eventual solution of MHD if an anomalous resistivity is locally enhanced around an X neutral point. Although the model is idealized, it is important to know why and how the fast reconnection mechanism can build up without any specific external agency. As shown in the paper, the sheet current is initially redistributed so as to avoid the localized diffusion region of enhanced resistivity. The underlying physics lies in the subsequent hydromagnetic stage, in which the resulting $\boldsymbol{J} \times \boldsymbol{B}$ force drives the plasma flow to effectively concentrate the current into the diffusion region. The remarkable reconnection dynamics results from the basic features of the current sheet system in collisionless plasmas.

On the basis of Ugai and Tsuda (1977), we have proposed the spontaneous fast reconnection model and demonstrated by 2-D and three-dimensional (3-D) MHD simulations that the fast reconnection mechanism can be realized by a nonlinear instability due to the positive feedback between currentdriven anomalous resistivities and global reconnection flows (Ugai, 1984, 1992, 1999; Ugai and Zheng, 2005). In the 3D current sheet system without initial sheared field, the key physical condition for the fast reconnection evolution is that the current sheet width in the sheet current direction is about three times larger than its thickness (Ugai, 2008a, 2009b). In fact, once such a thin current sheet is formed in collisionless plasmas, initiated by a reconnection disturbance, extreme current sheet thinning (current concentration) occurs

Published by Copernicus Publications on behalf of the European Geosciences Union. 


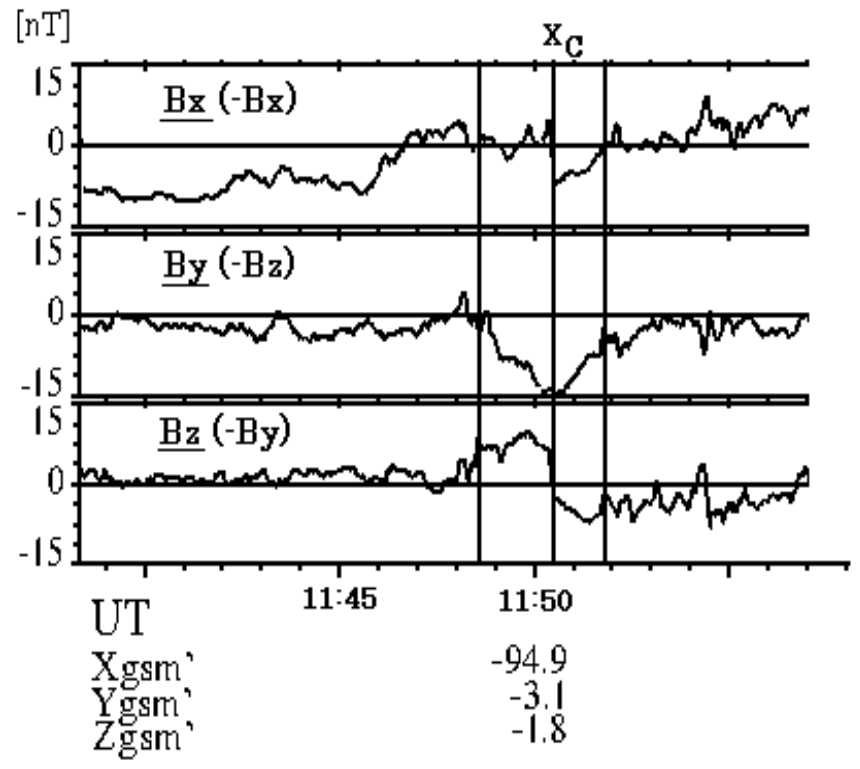

Fig. 1. Geotail satellite observations of magnetic fields of a plasmoid in the aberrated geocentric solar magnetosphere (GSM) coordinates, where the corresponding magnetic field components for the present numerical corrdinate system are shown in parentheses, and $X_{\mathrm{C}}$ indicates the plasmoid center (after Ieda et al., 1998).

around an $\mathrm{X}$ neutral line, which cannot be suppressed by MHD effects (Ugai, 1986, 2008a). It is hence reasonable to consider that the current sheet thinning drastically proceeds, until current-driven anomalous resistivities are necessarily caused in collisionless plasmas (Lui, 2001, 2004; Petkaki and Freeman, 2008). This is the reason why the fast reconnection evolution is little influenced by the functional form nor the parameter value of current-driven anomalous resistivity model (Ugai, 1984, 1992, 1999; Ugai and Zheng, 2005). In the presence of initial sheared field, the fast reconnection evolution becomes harder for the larger sheared field (Ugai, 2010a), and in a force-free current sheet the condition for the fast reconnection evolution is that the current sheet width is about six times larger than its thickness (Ugai, 2010b). In other words, the fast reconnection mechanism can readily build up spontaneously in such thin current sheets once they are formed in space (collisionless) plasmas.

Perhaps, the most typical observational signatures that provide clear evidences of magnetic reconnection may be those associated with plasmoids propagating down the geomagnetic tail during substorms. Historically, it has been believed that in accordance with the substorm onset a largescale plasmoid is generated because of the toplogical reconfiguration of magnetic field caused by explosive occurrence of magnetic reconnection. Hence, an essential question is to clarify how a large-scale plasmoid is generated by magnetic reconnection and propagates in the tail current sheet, and the key to understanding the plasmoid dynamics is to know the magnetic field topology inside the plasmoid. Figure 1 typi- cally shows the temporal changes in magnetic fields observed inside a plasmoid, which indicates that at the plasmoid center $X_{\mathrm{C}}$ the east-west field component $\left|B_{\mathrm{y}}\right|$ has the peak value and the north-south field component $B_{\mathrm{Z}}$ changes its sign (Ieda et al., 1998). These signatures of magnetic fields are usually observed inside plasmoids. Since the north-south field component changes its sign at the plasmoid center, it has commonly been accepted that the magnetic field lines inside the plasmoid are spiral or helical; otherwise, they are closed in a magnetic island. However, this conclution is based on satellite observations at limited spatial locations. In order to clarify the overall magnetic field topology of plasmoid, it is essential to fully understand the physical mechanism of plasmoid generation.

The plasmoid dynamics has been studied on the basis of the spontaneous fast reocnnection model by 2-D (Ugai, 1995) and 3-D MHD simulations (Ugai and Zheng, 2006a, b). In general, the plasmoid is generated as a result of drastic plasma compression ahead of the fast reconnection jet, and it swells and propagates along the antiparallel magnetic fields. Since the plasma pressure is notably enhanced inside the plasmoid, the plasmoid propagation may cause distinct signatures in the ambient magnetic field region outside the plasmoid. In fact, the so-called travelling compression regions (TCRs) have been observed in the geomagnetic tail lobe region. As well known, the TCR is considered to be a definite evidence of a plasmoid propagating down the central current sheet (Slavin et al., 1984, 1993). According to satellite observations, the pulse-like compression of the antiparallel field component $\left|B_{\mathrm{X}}\right|$ propagates down the tail, and at the TCR center the north-south and east-west magnetic field components have the bipolar structures. On the basis of the 3-D spontaneous fast reconnection model in the absence of initial sheared field, we demonstrated that these distinct magnetic field signatures in the ambient magnetic field region are the direct outcome of the plasmoid propagation, and the TCR signatures can exactly be explained both qualitatively and quantitatively (Ugai and Zheng, 2006a, b).

In order to understand the underlying physics of substorms, it is fundamental to clarify the magnetic field topology that is formed inside the plasmoid in accordance with the drastic occurrence of fast reconnection in the general sheared current sheet. Recently, we performed 3-D MHD simulations in a sheared current sheet, where a sheared field initially exists only in the central current sheet (Ugai, 2010a, b). It is demonstrated that the magnetic field profiles inside the plasmoid are generally consistent with satellite observations (Fig. 1), and the field lines inside the plasmoid are not spiral or helical. In the present study, the simulation model is extended to the general 3-D situation where sheared fields are initially present both outside and inside the current sheet, whereas any magnetic flux closure across the current sheet is not considered initially. The main theme of the present study is to further examine the magnetic field structure that is formed inside the large-scale plasmoid in the 
sheared current sheet. Here, it should be noted that $B_{\mathrm{x}}, B_{\mathrm{y}}$ and $B_{\mathrm{Z}}$ in the present coordinate system correspond to, respectively, $-B_{\mathrm{x}},-B_{\mathrm{z}}$ and $-B_{\mathrm{y}}$ in the conventional GSM coordinates (Fig. 1) (Ugai and Zheng, 2006a, b).

\section{Simulation modeling}

Except for the initial sheared field magnitude and the system length $L_{\mathrm{X}}$, the present simulation model is almost the same as Ugai (2010a). Also, all the details of the numerical procedure are already described in Ugai (2008a), which assures that throughout the present computations the numerical resistivity involved is much smaller than the physical (anomalous) resistivity. In fact, our numerical scheme is very simple and almost the same as the one employed more than 30 years before by Ugai and Tsuda (1977), and all our simulation results can readily be confirmed on the basis of Ugai (2008a).

\subsection{Basic equations}

The compressible MHD equations are

$D \rho / D t=-\rho \nabla \cdot \boldsymbol{u}, \quad \rho D \boldsymbol{u} / D t=-\nabla P+\boldsymbol{J} \times \boldsymbol{B}$,

$\partial \boldsymbol{B} / \partial t-\nabla \times(\boldsymbol{u} \times \boldsymbol{B})=-\nabla \times(\eta \boldsymbol{J})$,

$\rho D e / D t=-P \nabla \cdot \boldsymbol{u}+\eta \boldsymbol{J}^{2}$,

$\boldsymbol{J}=\nabla \times \boldsymbol{B} / \mu_{0}, \quad \nabla \cdot \boldsymbol{B}=0$,

where $D / D t \equiv \partial / \partial t+\boldsymbol{u} \cdot \nabla$; the gas law, $P=(\gamma-1) \rho e$, is assumed $[e$ is the internal energy per unit mass, and $\gamma$ is the specific heat ratio with $\gamma=5 / 3$ assumed here (an adiabatic case)], as is Ohm's law, $\boldsymbol{E}+u \times B=\eta \boldsymbol{J}$ ( $\eta$ may be an effective resistivity). The basic equations (1) are transformed to a conservation-law form, and the modified Lax-Wendroff scheme is used for the numerical computation (Ugai, 2008a).

\subsection{Initial-boundary conditions}

Initially, a long current sheet with sheared antiparallel magnetic fields $\left(B_{\mathrm{X}}\right)$ is assumed. Then, the normalization of quantities, based on the initial quantities, is self-evident: Distances are normalized by the y-directional current sheet halfthickness $d_{0}$, magnetic field $\boldsymbol{B}$ by $B_{x 0}$, plasma pressure $P$ by $P_{0}=B_{x 0}^{2} /\left(2 \mu_{0}\right)$; also, plasma density $\rho$ by $\rho_{i}=\rho(y=0)$, plasma flow velocity $\boldsymbol{u}$ by $V_{A x 0}\left(=B_{x 0} / \sqrt{\mu_{0} \rho_{i}}\right)$, time $t$ by $d_{0} / V_{A x 0}$. The Alfvén velocity based on $B_{x 0}$ in the ambient magnetic field region is given by $V_{A e}=V_{A x 0} / \sqrt{\rho_{e}}\left(\rho_{e}\right.$ is the plasma density in the magnetic field region).

The magnetic field $\boldsymbol{B}=\left[B_{\mathrm{X}}(y), 0, B_{\mathrm{Z}}(y)\right]$ is initially assumed as: $B_{\mathrm{x}}(y)=\sin (\pi y / 2)$ for $0<y<1 ; B_{\mathrm{x}}=1$ for $1<y<5 ; B_{\mathrm{x}}=\cos [(y-5) \pi / 0.6]$ for $5<y<5.3 ; B_{\mathrm{x}}=0$ for $y>5.3$; also, $B_{\mathrm{x}}(y)=-B_{\mathrm{X}}(-y)$ for $y<0$. The sheared field component $B_{\mathrm{Z}}$ is initially assumed as: $B_{\mathrm{Z}}(y)=B_{z 0}+$ $\alpha \cos (y \pi / 2)$ for $|y|<1$ and $B_{z}=B_{z 0}$ for $|y|>1$. Here, $B_{z 0}$ and $\alpha$ are taken to be parameters for the initial sheared field. Fluid velocity $\boldsymbol{u}=(0,0,0)$, and plasma pressure $P(y)$ satisfies the pressure-balance condition,

$P+B_{\mathrm{x}}^{2}+B_{\mathrm{z}}^{2}=1+\beta_{0}+B_{z 0}^{2}$,

where $\beta_{0}$ is the ratio of the plasma pressure to the magnetic pressure $B_{x 0}^{2} /\left(2 \mu_{0}\right)$ in the magnetic field region $1<y<5$ (here, $\beta_{0}=0.15$ is taken). Also, plasma density $\rho$ initially satisfies

$\rho(y)=P(y) /\left(1+\beta_{0}+B_{z 0}^{2}-\left(B_{z 0}+\alpha\right)^{2}\right)$,

so that $\rho_{e}=\beta_{0} /\left(1+\beta_{0}+B_{z 0}^{2}-\left(B_{z 0}+\alpha\right)^{2}\right)$ in the ambient magnetic field region, and the initial temperature $T=P / \rho$ is assumed to be constant everywhere.

In our previous studies on 3-D plasmoids, the conventional plane symmetry boundary conditions were assumed on the $z=0, x=0$ and $y=0$ planes, and the computational region was restricted to the first quadrant only (Ugai and Zheng, 2006a, b). Here, the computational region is extended to the second quadrant in the $z$ direction and is taken to be a rectangular box, $0 \leq x \leq$ $L_{x}, 0 \leq y \leq L_{y}$, and $-L_{z} \leq z \leq L_{z}$, and the axis symmetry boundary conditions are assumed as follows (Ugai, 2010a). With respect to the $\mathrm{x}$ axis, $\rho(x,-y,-z)=$ $\rho(x, y, z), \quad u_{x}(x,-y,-z)=u_{x}(x, y, z), \quad u_{y}(x,-y,-z)=$ $-u_{y}(x, y, z), u_{z}(x,-y,-z)=-u_{z}(x, y, z), B_{x}(x,-y,-z)=$ $-B_{x}(x, y, z), B_{y}(x,-y,-z)=B_{y}(x, y, z), B_{z}(x,-y,-z)=$ $B_{z}(x, y, z)$, and $P(x,-y,-z)=P(x, y, z)$, for a fixed value of $x$ in $0 \leq x \leq L_{x}$. With respect to the $y$ axis, $\rho(-x, y,-z)=\rho(x, y, z), \quad u_{x}(-x, y,-z)=-u_{x}(x, y, z)$, $u_{y}(-x, y,-z)=u_{y}(x, y, z), u_{z}(-x, y,-z)=-u_{z}(x, y, z)$, $B_{x}(-x, y,-z)=B_{x}(x, y, z), B_{y}(-x, y,-z)=-B_{y}(x, y, z)$, $B_{z}(-x, y,-z)=B_{z}(x, y, z)$, and $P(-x, y,-z)=P(x, y, z)$, for a fixed value of $y$ in $0 \leq y \leq L_{y}$. On the other boundary planes $\left(x=L_{x}, y=L_{y}, z=-L_{z}\right.$ and $\left.z=L_{z}\right)$, the free boundary conditions are assumed, so that the reconnection ouflow is open to the free space in the positive $\mathrm{x}$-direction.

The effective resistivity for the Ohm's law (normalized by $\left.\mu_{0} d_{0} V_{A x 0}\right)$ is assumed to be,

$$
\begin{aligned}
\eta(\mathbf{r}, t) & =k_{d}\left[V_{d}(\mathbf{r}, t)-V_{\mathrm{C}}\right] \quad \text { for } V_{d}>V_{\mathrm{C}}, \\
& =0 \quad \text { for } V_{d}<V_{\mathrm{C}},
\end{aligned}
$$

where $V_{d}(\mathbf{r}, t)=|\boldsymbol{J}(\mathbf{r}, t) / \rho(\mathbf{r}, t)|$ is the relative electron-ion drift velocity. Here, we take $k_{d}=0.003$ and $V_{\mathrm{C}}=12$ in Eq. (4) as before. Also, in order to disturb the initial configuration, a localized resistivity is assumed around the origin $(\mathbf{r}=0)$ in the $3-\mathrm{D}$ form,

$\eta(\mathbf{r})=\eta_{0} \exp \left[-\left(x / k_{x}\right)^{2}-\left(y / k_{y}\right)^{4}-\left(z / k_{z}\right)^{4}\right]$,

which causes magnetic reconnection as an initial disturbance. Here, we take $\eta_{0}=0.02$ and $k_{x}=k_{y}=0.8$, and $k_{z}=5$. This disturbance (5) is imposed only in the initial time range $0<t<4$, and the resistivity model (4) is assumed for $t>4$. 


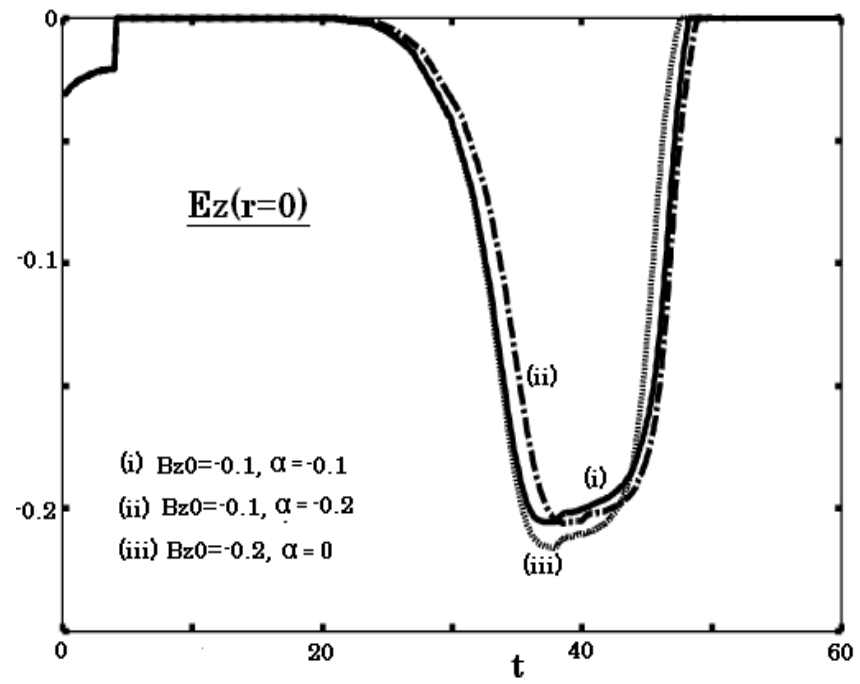

Fig. 2. Temporal variations in the electric field $E_{\mathrm{Z}}(\mathbf{r}=0)$ at the origin for different values of $B_{z 0}$ and $\alpha$.

The parameters for the numerical computations are taken to be $L_{x}=20, L_{y}=7.2$, and $L_{z}=10$ (in Ugai, 2010a, $L_{x}=$ 14 was taken). The mesh sizes are $\Delta x=0.04, \Delta y=0.015$ and $\Delta z=0.1$. As was shown in detail in Ugai (2008a), the numerical resistivity for these parameters is about 0.0005 , which is found to be much smaller than the physical (anomalous) resistivity throughout the present computations.

\section{Results}

In Ugai (2010a, b), MHD simulations were done for different values of $\alpha$ with $B_{z 0}=0$ being fixed, and the fast reconnection evolution in a force-free current sheet was examined. Here, we consider the more general case of nonzero $B_{z 0}$ outside the current sheet, and computations are done typically for the three cases, (i) $B_{z 0}=-0.1$ and $\alpha=-0.1$, (ii) $B_{z 0}=-0.1$ and $\alpha=-0.2$, and (iii) $B_{z 0}=-0.2$ and $\alpha=0$. In each case, the sheared field is rather small, so that the fast reconnection mechanism is found to fully evolve for $k_{z}=5$ in Eq. (5) (Ugai, 2010a, b). The basic physical processes of the plasmoid generation in the absence of sheared field were already shown in detail in the 2-D (Ugai, 1995) and 3-D (Ugai and Zheng, 2006a, b) situations. In what follows, the general features of the fast reconnection evolution is first summarized briefly, and then the magnetic field profiles associated with the sheared plasmoid are examined in detail.

\subsection{General remarks}

We find that the fast reconnection evolution is not significantly influenced by the parameter $B_{z 0}$ or $\alpha$. Figure 2 shows the temporal variations in the electric field $E_{\mathrm{Z}}$ at the origin for

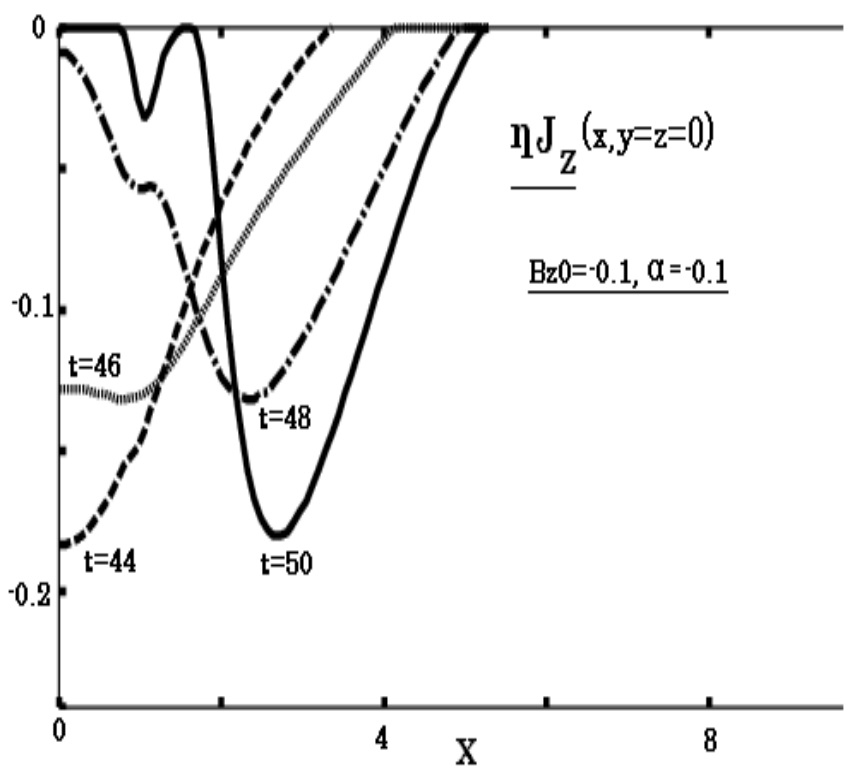

Fig. 3. Profiles of $\eta J_{\mathrm{Z}}$ along the $\mathrm{x}$-axis for the case of $B_{z 0}=-0.1$ and $\alpha=-0.1$ at different times.

different cases of $B_{z 0}$ and $\alpha$. In each case, initiated by the initial disturbance (5) in the time range $0<t<4$, the resulting $\boldsymbol{J} \times \boldsymbol{B}$ force drives the reconnection flows so as to concentrate the current around the origin for $t>4$. Accordingly, the threshold of the anomalous resistivity in Eq. (4) is eventually exceeded at $t \sim 24$, so the fast reconnection mechanism drastically evolves because of the positive feedback between the reconnection flow and the anomalous resistivity to attain the reconnection electric field $\left|E_{\mathrm{Z}}\left(=\eta J_{\mathrm{Z}}\right)\right| \sim 0.2$ (normalized by $\left.V_{A x 0} B_{x 0}\right)$ at time $t \sim 36$ on the nonlinear saturation phase. The fast reconnection proceeds almost steadily for a while, until the secondary tearing suddenly occurs at $t \sim 46$, so the $\mathrm{X}$ neutral line is shifted in the $\mathrm{X}$-direction and the origin becomes an $\mathrm{O}$ neutral line. Figure 3 shows the profiles of $\eta J_{Z}$ along the $\mathrm{x}$-axis at different times typically for the case of $B_{z 0}=-0.1$ and $\alpha=-0.1$. At times $t=44$ and 46 the diffusion region becomes longer in the $\mathrm{x}$-direction, and at $t=48$ the location of the peak value of $\eta J_{\mathrm{z}}$, where the $\mathrm{X}$ neutral line is located, shifts in the $\mathrm{x}$-direction and the peak value recovers to $\sim-0.2$ at $t \sim 50$. The magnetic island formed at the origin swells with time but cannot propagate because of the present symmetry boundary conditions.

Figure 4 shows the magnetic field lines projected onto the $z=0$ plane at different times for the case of $B_{z 0}=-0.1$ and $\alpha=-0.1$; also, Fig. 5 shows the corresponding distributions of the plasma pressure $P$ in the $z=0$ plane. At $t=36$, the fast reconnection mechanism has just built up, and the $X$ field configuration is formed around the $\mathrm{X}$ neutral line located at the origin $(\mathbf{r}=0)$. At $t=41$ the fast reconnection mechanism is fully established, and the overall configuration can be divided into the diffusion region (in $|x|<X_{\mathrm{D}}$ ), the 

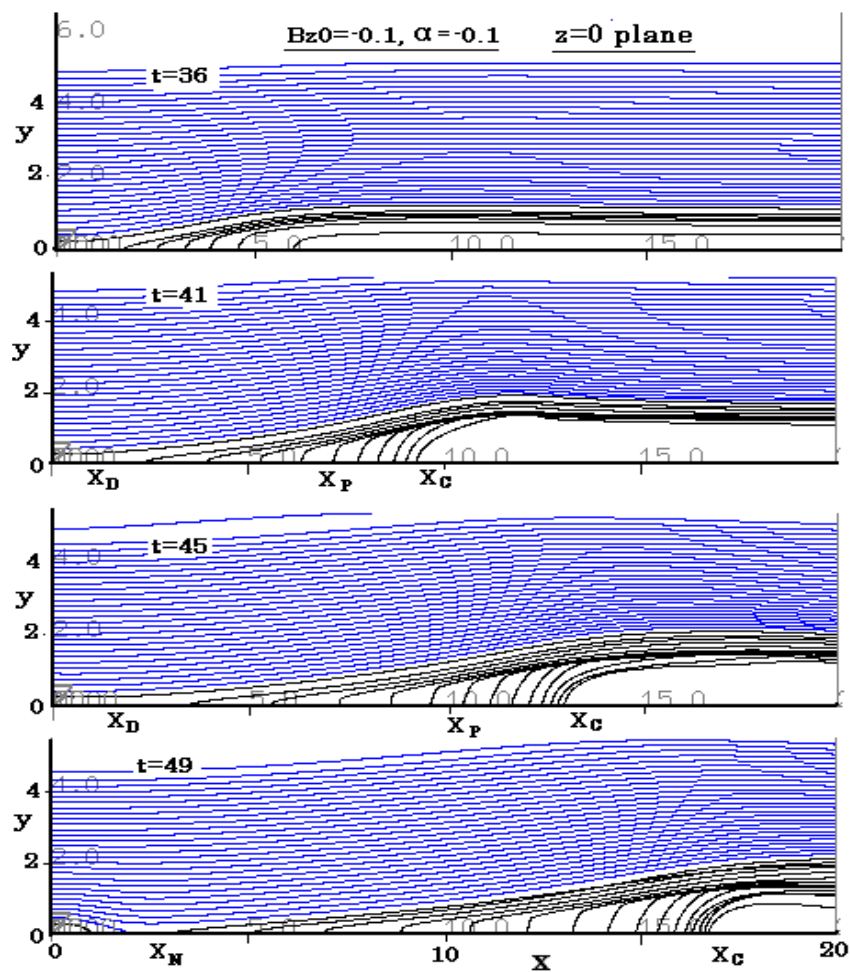

Fig. 4. Magnetic field lines passing through the y-axis and the reconnected field lines passing through the $\mathrm{x}$-axis, projected onto the $z=0(x, y)$ plane, for the case of $B_{z 0}=-0.1$ and $\alpha=-0.1$, where the diffusion region boundary $X_{\mathrm{D}}$, the plasmoid backward end $X_{\mathrm{P}}$ and the plasmoid center $X_{\mathrm{C}}$ are indicated at $t=41$ and 45; also, the shifted $X$ neutral line $X_{\mathrm{N}}$ is indicated at $t=49$.

fast reconnection jet region (in $X_{\mathrm{D}}<x<X_{\mathrm{P}}$ ), and the largescale plasmoid region, where $X_{\mathrm{D}}$ and $X_{\mathrm{P}}$ are, respectively, the boundary of the diffusion region and the backward end of the plasmoid. In the diffusion region, the anomalous resistivity remains to be locally enhanced, and in the fast reconnection jet region, the plasma outflow velocity is accelerated to the Alfvén velocity $V_{A e}$ by standing slow shocks (Ugai, 2010a). The large-scale plasmoid, which is generated ahead of the Alfvénic jet by the drastic plasma compression, swells with time and propagates in the positive $\mathrm{x}$-direction. Inside the plasmoid, the enhanced plasma pressure is stored and the sheared fields are accumulated. At $t \sim 46$ the secondary tearing suddenly occurs, so at $t=49$ the $\mathrm{X}$ neutral line $X_{\mathrm{N}}$ shifts in the $\mathrm{X}$-direction and a magnetic island is formed around the origin.

More quantitatively, Fig. 6 shows the profiles of the plasma pressure $P$, the outflow velocity $u_{x}$, and the magnetic field components $B_{\mathrm{y}}$ and $B_{\mathrm{z}}$ along the x-axis for the case of $B_{z 0}=-0.1$ and $\alpha=-0.1$. At $t=41$, the outflow velocity $u_{x}$ in the fast reconnection jet region attains the Alfvén velocity $V_{A e}$ (Ugai, 2010a), measured in the ambient magnetic field region, and $u_{x}$ is suddenly decelerated at the plasmoid backward end $X_{\mathrm{P}}$ so as to compress the magnetized
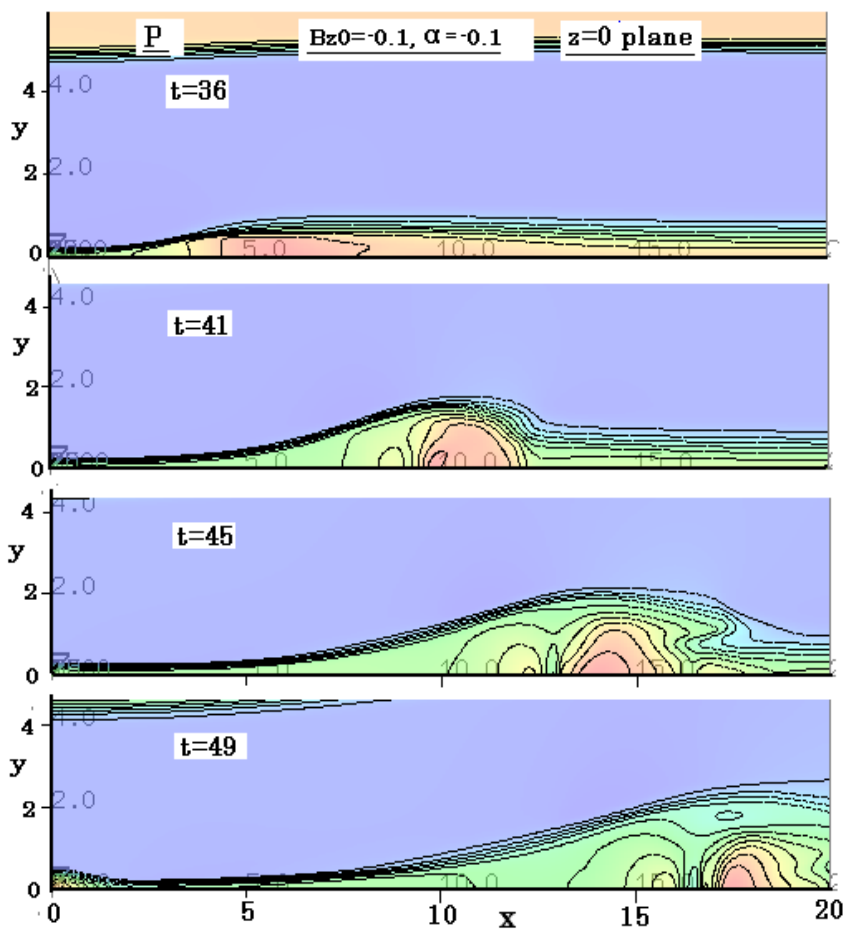

Fig. 5. Distributions of plasma pressure $P$ with the contour interval of 0.15 in the $z=0$ plane at different times for the case of $B_{z 0}=$ -0.1 and $\alpha=-0.1$.

plasma. Accordingly, the sheared magnetic field $B_{\mathrm{Z}}$ is significantly compressed and has the peak value at the plasmoid center $x=X_{\mathrm{C}}$, which is defined as the location where the $B_{\mathrm{y}}$ field changes its sign (Ugai, 2010a, b). As time progresses, the fast reconnection jet region at $t=45$ extends in the positive $\mathrm{x}$-direction, leading to the plasmoid propagation. Inside the plasmoid, the reconnected field lines with $B_{\mathrm{y}}>0$ are piled up in $X_{\mathrm{P}}<x<X_{\mathrm{C}}$, and the sheared field lines, initially residing in the central current sheet, has been swept away by the reconnection jet $u_{x}$ and are accumulated in $X_{\mathrm{C}}<x$. Hence, the location of $x=X_{\mathrm{C}}$ may correspond to the socalled contact discontinuity that bounds the reconnected and non-reconnected field lines. For the other parameter values, we find that the basic profiles of these quantities are little infulenced by $B_{z 0}$ or $\alpha$.

\subsection{Magnetic field profiles of the plasmoid}

On the basis of the resulting large-scale plasmoid, the general features of the magnetic field profiles around the plasmoid center may directly be examined. Since the basic plasmoid structure does not significantly depend on the parameter $B_{z 0}$ or $\alpha$, mainly the typical case of $B_{z 0}=-0.1$ and $\alpha=-0.1$ will be shown. Figure 7 shows the plasma flow vectors in the $z=0$ plane at $t=41$ and 45 ; also, the magnetic field lines passing through the $\mathrm{y}$-axis and the reconnected field lines passing through the $\mathrm{x}$-axis are shown at $t=45$, where the 

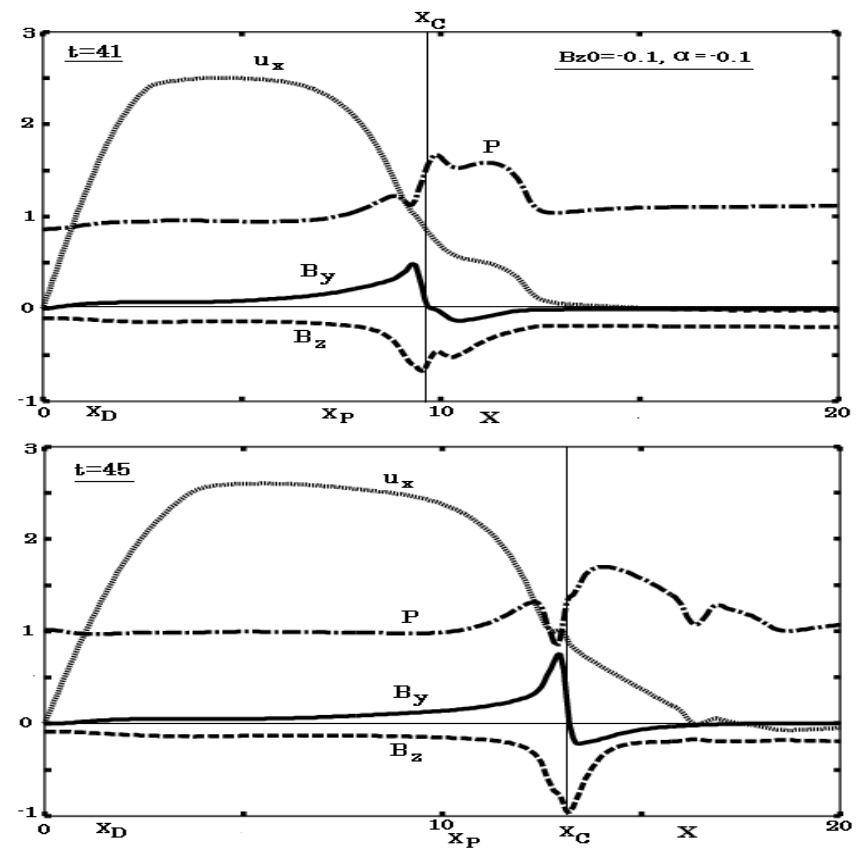

Fig. 6. Profiles of magnetic field components $B_{\mathrm{y}}$ and $B_{\mathrm{z}}$, plasma pressure $P$, and the outflow velocity $u_{x}$ along the $\mathrm{x}$-axis at times $t=41$ and 45 for the case of $B_{z 0}=-0.1$ and $\alpha=-0.1$, where the diffusion region boundary $X_{\mathrm{D}}$, the plasmoid backward end $X_{\mathrm{P}}$ and the plasmoid center $X_{\mathrm{C}}$ are indicated.

isosurface of plasma pressure $P=1.5$, inside which $P>1.5$ (initially, $P=1.12$ at $y=0$ for this case), is also shown. The fast reconnection jet region with $u_{x} \sim V_{A e}$ (Fig. 6) extends with time in the positive x-direction. Ahead of the reconnected field lines, the magnetized plasma initially in the current sheet has been distinctly compressed, so that the plasma pressure is notably enhanced in $x>X_{\mathrm{C}}$ (Fig. 6). We readily see that the reconnected field lines are not spiral or helical (Fig. 4), but they are simply skewed in the sheared field direction. Also, Fig. 8 shows the distributions of the field components $B_{\mathrm{x}}, B_{\mathrm{y}}$ and $B_{\mathrm{Z}}$ in the $z=0$ plane at times $t=41$ and 45. Because of the symmetry boundary conditions, $B_{\mathrm{x}}=0$ along the $\mathrm{x}$-axis and $B_{\mathrm{y}}=0$ along the $\mathrm{y}$-axis. Around the plasmoid center $x \sim X_{\mathrm{C}}, B_{\mathrm{y}}$ changes its sign and $\left|B_{\mathrm{Z}}\right|$ has the peak value. Also, the $B_{\mathrm{X}}$ field is notably compressed locally at $x \sim X_{\mathrm{C}}$ inside the plasmoid.

Since the plasmoid propagates in the positive $\mathrm{x}$-direction, it is instructive to see the $\mathrm{x}$-directional profiles of magnetic fields around the plasmoid center. Figure 9 shows at $t=41$ and 45 the profiles of the field components $B_{\mathrm{x}}, B_{\mathrm{y}}$ and $B_{\mathrm{Z}}$ in the $\mathrm{x}$-direction at $y=0.15$ and $z=-1$ near the $\mathrm{x}$-axis for the case of $B_{z 0}=-0.1$ and $\alpha=-0.1$. As already stated, the plasmoid center $X_{\mathrm{C}}$ may be defined as the position where $B_{\mathrm{y}}$ changes its sign. We see that at $x=X_{\mathrm{C}}\left|B_{\mathrm{Z}}\right|$ has the peak value, and changes in $B_{\mathrm{x}}$ around $X_{\mathrm{C}}$ are similar to those of $B_{\mathrm{y}}$; in particular, at $t=45 B_{\mathrm{x}}$ as well as $B_{\mathrm{y}}$ changes its sign at $x=X_{\mathrm{C}}$ in the same manner. Similarly, Fig. 10 shows the
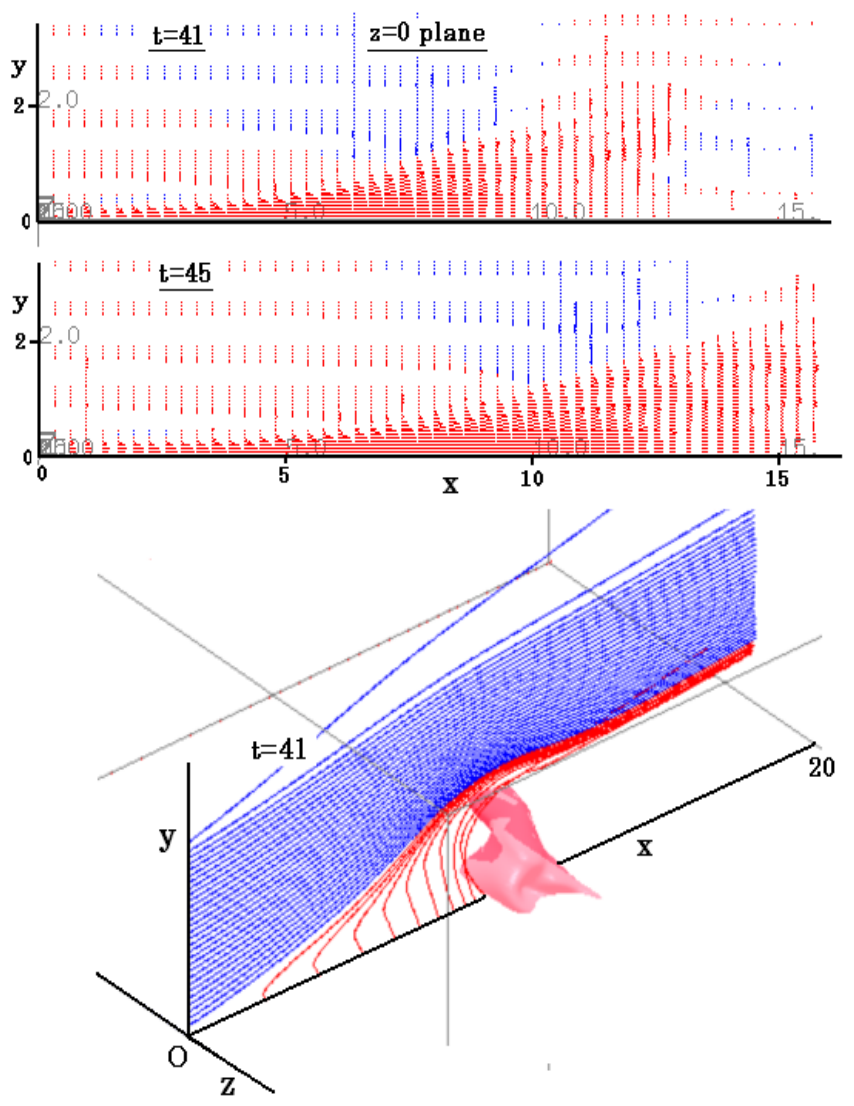

Fig. 7. Upper panel: plasma flow velocity vectors projected onto the $z=0$ plane at $t=41$ and 45; lower panel: magnetic field lines passing through the $y$-axis and the reconnected field lines passing through the $\mathrm{x}$-axis at $t=45$, where the isosurface of plasma pressure $P=1.5$ is also shown $\left(B_{z 0}=-0.1\right.$ and $\left.\alpha=-0.1\right)$.

$\mathrm{x}$-directional profiles of the field components at $y=0.15$ and $z=1.0$ in the positive $z$ side. In this case, the profiles of $B_{\mathrm{y}}$ and $B_{\mathrm{z}}$ are simiar to those in Fig. 9, whereas the profile of $B_{\mathrm{X}}$ around $x=X_{\mathrm{C}}$ seems to be somewhat shifted and not fully coincident with the $B_{\mathrm{y}}$ profile. Also, Fig. 11 shows the magnetic field profiles at $t=45$ at larger distances from the $\mathrm{x}$-axis in the $\mathrm{z}$-direction. The profiles at $z=2$ and $z=-2$ are qulitatively similar to those at $z=1$ (Fig. 10) and $z=-1$ (Fig. 9), respectively, although changes in quantities become smaller at larger distances from the $\mathrm{x}$-axis. In general, the profiles of magnetic fields in $z<0$ (or $z>0$ ) are qualitatively similar, and in each case we find that the sheared field component $\left|B_{\mathrm{Z}}\right|$ has the peak value at $x=X_{\mathrm{C}}$, where $B_{\mathrm{y}}$ changes its sign.

We find that the basic profiles of magnetic fields inside the plasmoid are not infulenced by the parameter, $B_{z 0}$ or $\alpha$. In fact, we already reported that for the cases of $B_{z 0}=0$ and different values of $\alpha$ too, $\left|B_{Z}\right|$ has the peak value at the plasmoid center $x=X_{\mathrm{C}}$, where $B_{\mathrm{y}}$ changes its sign (Ugai, 2010a, b). In the manner similar to Fig. 9, Fig. 12 shows 

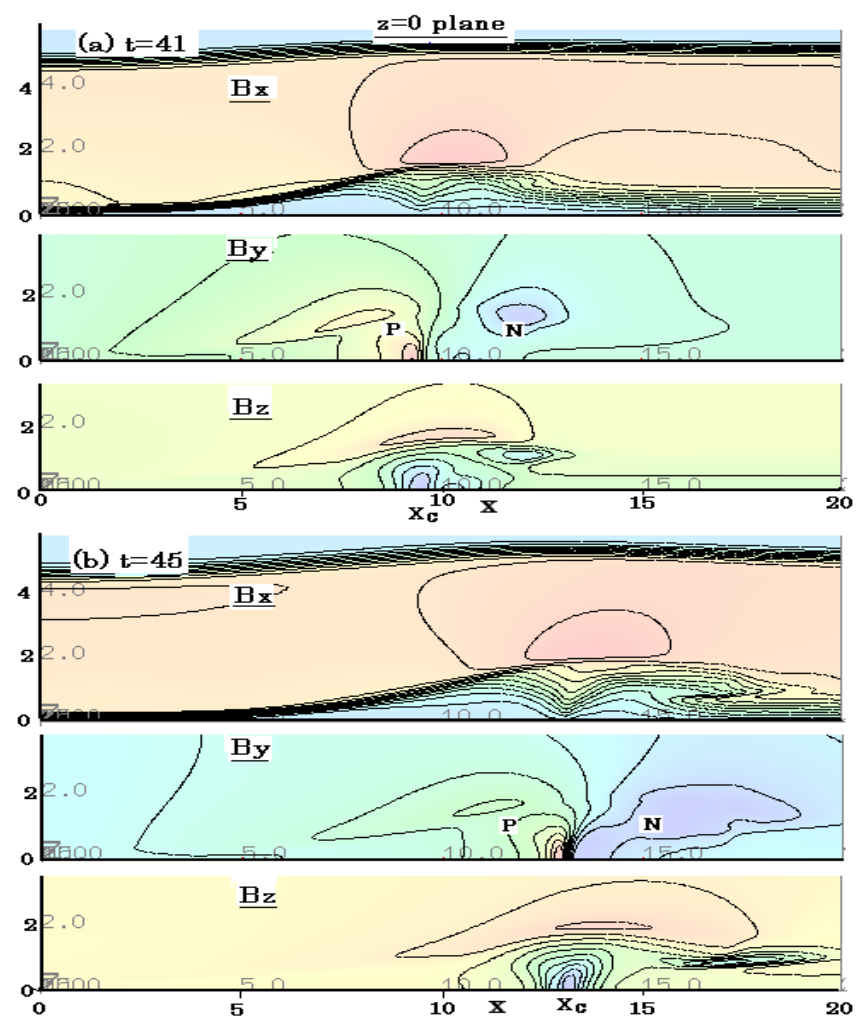

Fig. 8. Distributions of magnetic field components $B_{\mathrm{X}}, B_{\mathrm{y}}$ and $B_{\mathrm{Z}}$ in the $z=0$ plane at times $t=41$ and 45 with the contour interval of 0.1 , where the locations of the plasmoid center $X_{\mathrm{C}}$ is indicated, and $P$ and $N$ in $B_{\mathrm{y}}$ indicate, respectively, the positive value (the reconnected field lines) and negative value (the sheared field lines initially embedded in the current sheet $)\left(B_{z 0}=-0.1\right.$ and $\left.\alpha=-0.1\right)$.

the profiles of the field components $B_{\mathrm{x}}, B_{\mathrm{y}}$ and $B_{\mathrm{z}}$ in the $x$ direction at $y=0.15$ and $z=-1$ near the $\mathrm{x}$-axis for the case of $B_{z 0}=-0.1$ and $\alpha=-0.2$. The profiles are shown in the negative $z$ side at $t=44$ and 48 , when the fast reconnection mechanism is fully established (Fig. 2). Obviously, the $\mathrm{x}$-directional changes in magnetic fields inside the plasmoid are consistent with those in Fig. 9; in particular, at $t=48$ the $B_{\mathrm{x}}$ field component changes its sign at $x=X_{\mathrm{C}}$ in the same manner as $B_{\mathrm{y}}$. For the case of $B_{z 0}=-0.2$ and $\alpha=0$ too, we find that the results are quite similar. Hence, the magnetic field profiles inside the plasmoid have the general features independently of the parameter $B_{z 0}$ or $\alpha$. For instance, in the $z<0$ side for the present case of $B_{z}<0$, the $B_{\mathrm{y}}$ field changes its sign and the $\left|B_{\mathrm{Z}}\right|$ field has the peak value at the plasmoid center $x=X_{\mathrm{C}}$, and the profile of $B_{\mathrm{x}}$ coincides with that of $B_{\mathrm{y}}$ around $x=X_{\mathrm{C}}$.

Another well-known and distinct observational signature of a large-scale plasmoid is the TCR observed in the tail lobe region. We already demonstrated that the large-scale plasmoid generated by the fast reconnection evolution is exactly consistent with the satellite observations of TCRs both qualitatively and qunatitatively (Ugai and Zheng, 2006a, b).

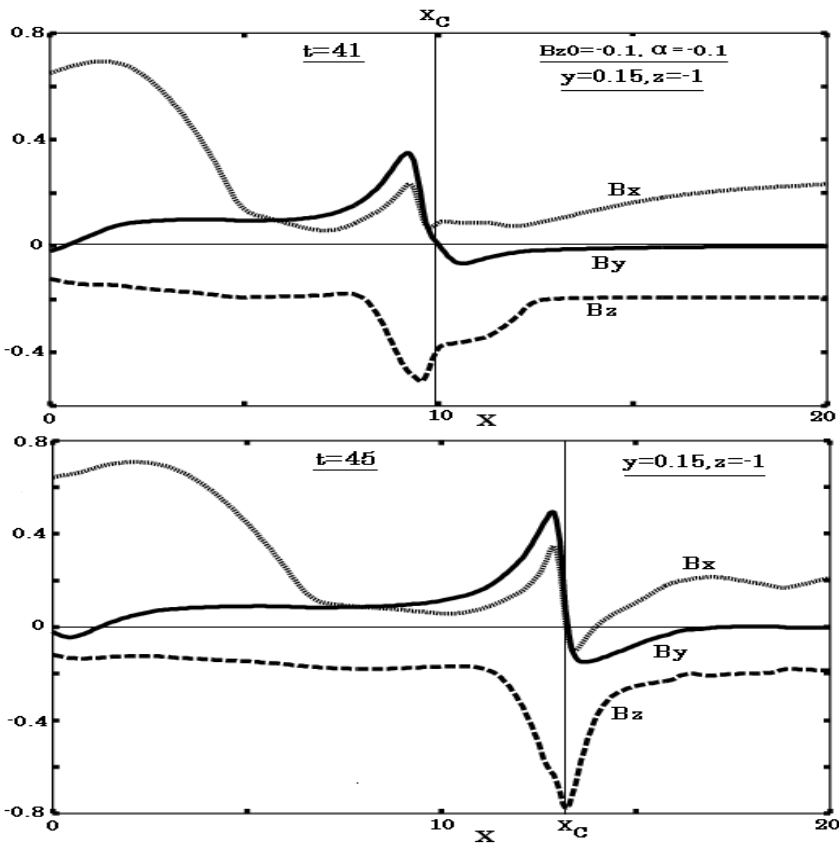

Fig. 9. x-directional profiles of magnetic field components $B_{\mathrm{x}}, B_{\mathrm{y}}$ and $B_{\mathrm{Z}}$ at $y=0.15$ and $z=-1.0$ at times $t=41$ and 45 for the case of $B_{z 0}=-0.1$ and $\alpha=-0.1$, where the plasmoid center $X_{\mathrm{C}}$ is indicated.

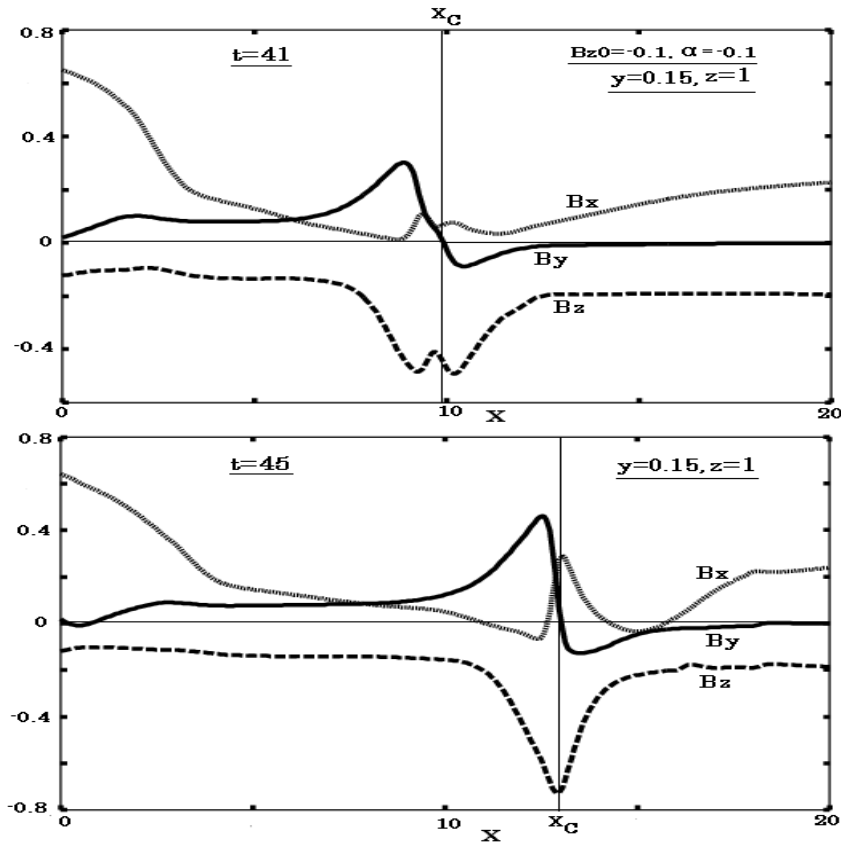

Fig. 10. x-directional profiles of magnetic field components $B_{\mathrm{x}}, B_{\mathrm{y}}$ and $B_{\mathrm{Z}}$ at $y=0.15$ and $z=1.0$ at times $t=41$ and 45 for the case of $B_{z 0}=-0.1$ and $\alpha=-0.1$, where the plasmoid center $X_{\mathrm{C}}$ is indicated. 

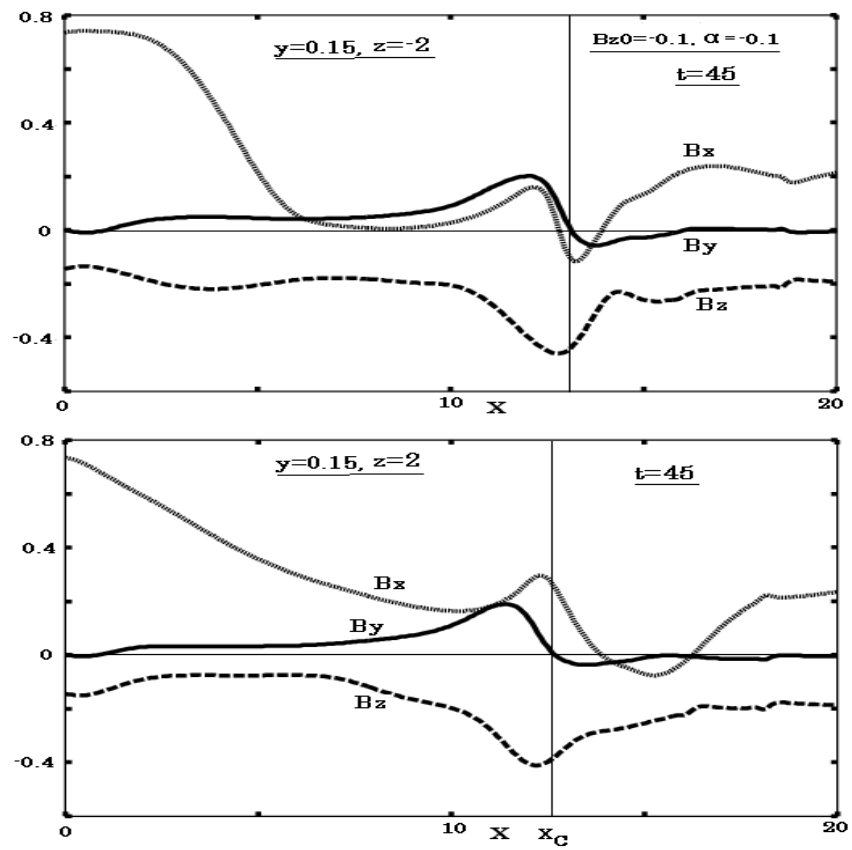

Fig. 11. x-directional profiles of magnetic field components $B_{\mathrm{X}}, B_{\mathrm{y}}$ and $B_{\mathrm{Z}}$ at $y=0.15$ and $z=-2.0$ and $z=2$ at time $t=45$ for the case of $B_{z 0}=-0.1$ and $\alpha=-0.1$, where the plasmoid center $X_{\mathrm{C}}$ is indicated.
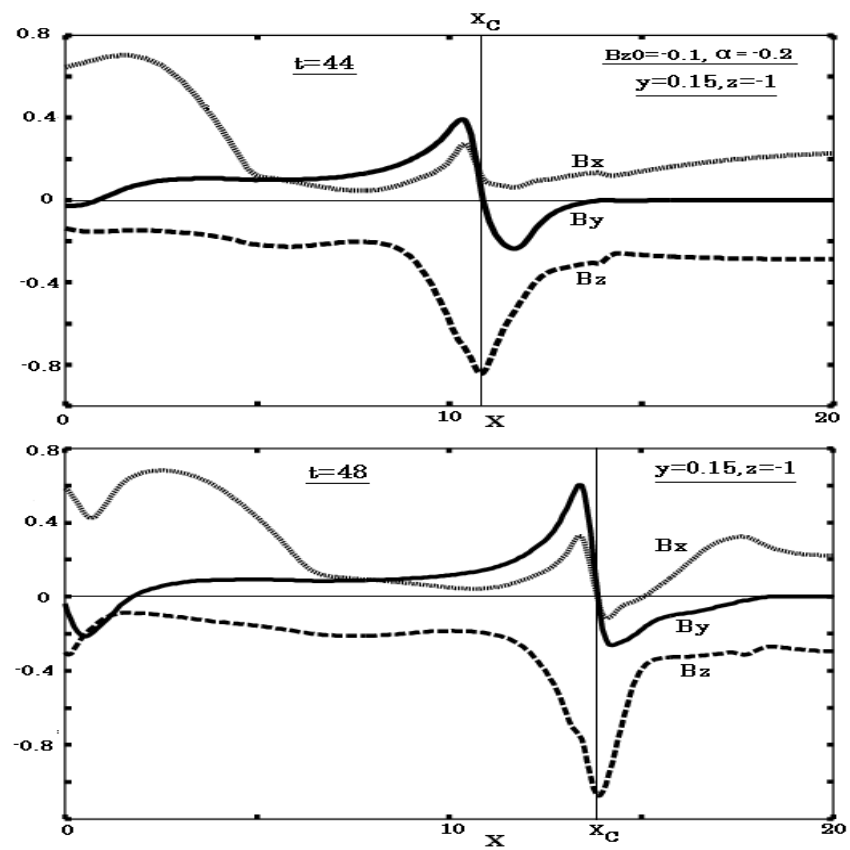

Fig. 12. x-directional profiles of magnetic field components $B_{\mathrm{x}}, B_{\mathrm{y}}$ and $B_{\mathrm{Z}}$ at $y=0.15$ and $z=-1.0$ at times $t=44$ and 48 for the case of $B_{z 0}=-0.1$ and $\alpha=-0.2$, where the plasmoid center $X_{\mathrm{C}}$ is indicated.
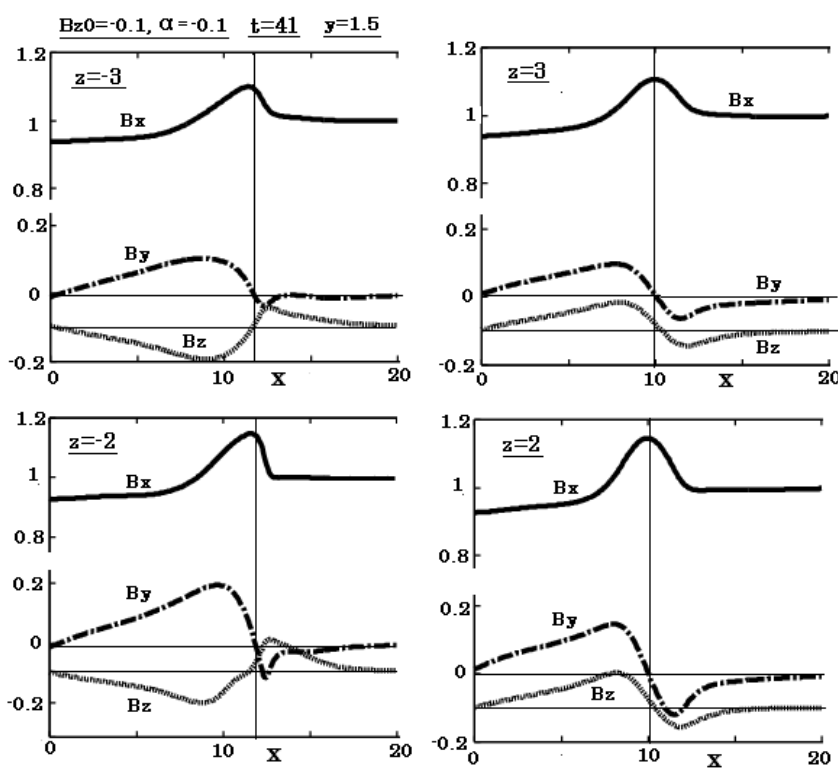

Fig. 13. x-directional profiles of magnetic field components $B_{\mathrm{x}}, B_{\mathrm{y}}$ and $B_{\mathrm{Z}}$ at different locations of $z$ at $y=1.5$ at time $t=41$ for the case of $B_{z 0}=-0.1$ and $\alpha=-0.1$.

However, these studies were performed for the fast reconnection in the absence of initial sheared field $\left(B_{z 0}=\alpha=0\right)$, so it is important to examine TCR signatures for the present sheared plasmoid configurations. In Fig. 8, the pulse-like compression of the $B_{\mathrm{X}}$ field above the plasmoid propagates in the positive $\mathrm{x}$-direction, consistent with the TCR. Here, the results for the case of $B_{z 0}=-0.1$ and $\alpha=-0.1$ may typically be examined, since the basic TCR signatures are found to be little influenced by the parameter values. Figure 13 shows the x-directional profiles of $B_{\mathrm{x}}, B_{\mathrm{y}}$ and $B_{\mathrm{z}}$, measured at $y=1.5$ outside the plasmoid at different $z$ locations at time $t=41$, when the overall plasmoid structure is found to be inside the computational domain. In each case, there is a pulselike compression of $B_{\mathrm{x}}$, and the field components $B_{\mathrm{y}}$ and $B_{\mathrm{z}}$ have the bipolar changes about their background values. The bipolar change in $B_{\mathrm{Z}}$ is opposite in the $z<0$ and $z>0$ sides. That is, for $z<0 B_{\mathrm{Z}}$ changes from negaive to positive values, whereas for $z>0$ it changes from positive to negative values. This is because the plasmoid expands both in the positive and negative $\mathrm{z}$-directions with respect to the $\mathrm{x}$-axis $(z=0)$. These featrures of magnetic field profiles in the ambient magnetic field region outside the plasmoid are in good agreement with the well-known TCR signatures obtained by satellite observations (Slavin et al., 1993).

\section{Summary and discussion}

On the basis of the spontaneous fast reconnection model, the 3-D dynamics of a large-scale plasmoid is studied for different parameter values of initial sheared magnetic field $\left(B_{\mathrm{Z}}\right)$ 


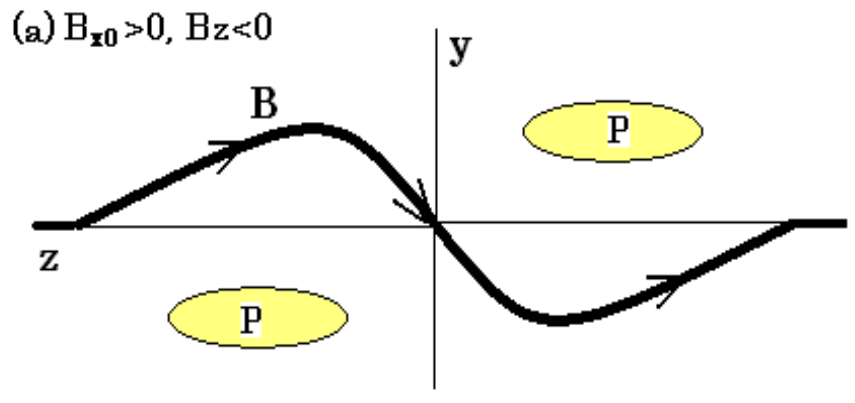

(b) $\mathrm{B}_{\mathbf{x} 0}>0, \mathrm{Bz}>0$

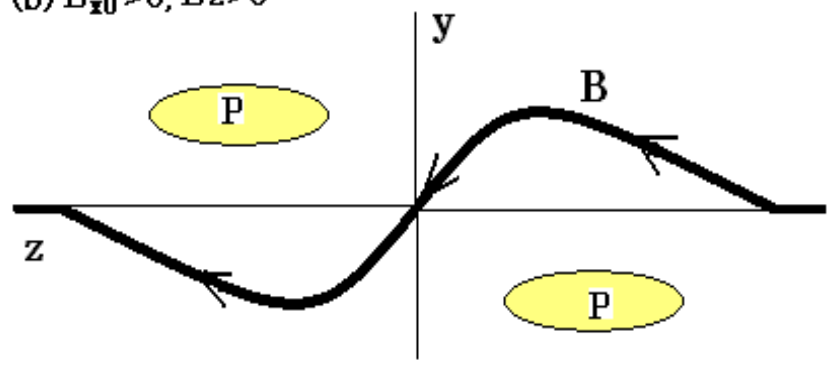

Fig. 14. Schematic drawings of the sheared field lines (solid lines) and the cross section of the high-pressure part of the distorted plasmoid, denoted by $P$, in the $(y, z)$ plane at a fixed location of $x\left(>X_{\mathrm{C}}\right)$ for the cases (a) $B_{x 0}>0$ and $B_{\mathrm{Z}}<0$, and (b) $B_{x 0}>0$ and $B_{\mathrm{Z}}>0$.

in the long current sheet system. The basic features of the plasmoid evolution are, at least qualitatively, not influenced by the initial sheared field. That is, the plasmoid is formed as a result of the drastic plasma compression at the plasmoid backward end at $x=X_{\mathrm{P}}$ ahead of the fast (Alfvénic) reconnection jet. It propagates along the antiparallel magnetic fields and its propagation tends to be distorted in the direction of the sheared field. Inside the plasmoid, the magnetized plasmas, which have been swept away by the fast reconnection outflow jet $u_{x}$, are accumulated. The magnetic field lines that have been piled up inside the plasmoid are composed of the two types topologically different. The sheared field lines accumulated in $X_{\mathrm{P}}<x<X_{\mathrm{C}}$ are the field lines that have been reconnected at the $X$ neutral line, whereas in $X_{\mathrm{C}}<x$ the sheared field lines initially embedded in the central current sheet are accumulated in the form of a core (Fig. 8). Hence, the plasmoid center $x=X_{\mathrm{C}}$ is the position that bounds the reconnected field lines and the field lines without reconnection, so it may correspond to the so-called contact discontinuity. Accordingly, the reconnected field lines are not spiral or helical, since the field lines in $x<X_{\mathrm{C}}$ are never connected topologically to those in $x>X_{\mathrm{C}}$ (Figs. 4 and 7).

A distinct feature of the magnetic field profiles inside the plasmoid is that the $B_{\mathrm{y}}$ field component becomes negative for $x>X_{\mathrm{C}}$ and hence changes its sign at $x=X_{\mathrm{C}}$, where the accumulated sheared field $\left|B_{\mathrm{Z}}\right|$ has the peak value (Figs. 6 and 8); also, the negative value of $B_{\mathrm{y}}$ for $x>X_{\mathrm{C}}$ tends to be- come more distinct for the larger sheared field accumulation (Figs. 9, 10 and 12). The similar results are recently obtained by 3-D MHD simulations, where a localized fixed resistivity model is assumed in the similar manner as Ugai and Tsuda (1977) (Shirataka et al., 2008). This does not mean that the reconnected field lines are closed (or spiral) in a flux rope. As already shown in Ugai (2010b), this is because the plasmoid propagation tends to be distorted in the direction of the sheared field. Figure 14 schematically illustrates this situation in the $(y, z)$ plane at a fixed location of $x\left(>X_{\mathrm{C}}\right)$ across the plasmoid. For the case of the accumulated sheared field $B_{\mathrm{z}}<0$, as shown in Fig. 14a, the plasmoid of high pressure is shifted in the negative $\mathrm{z}$-direction in $y>0$ (Fig. 7), which tends to push the sheared field lines $\left(B_{\mathrm{Z}}\right)$ downward (in the negative $y$-direction). On the other hand, in $y<0$ the plasmoid is shifted in the positive $\mathrm{z}$-direction because of the X-axis symmetry, which tends to push the $B_{\mathrm{Z}}$ field lines upward (in the positive $y$-direction). For the case of $B_{\mathrm{z}}>0$, as shown in Fig. 14b, the plasmoid distortion in the sheared field direction push the accumulated $B_{\mathrm{Z}}$ field lines in the similar manner. In each case, the region of $B_{\mathrm{y}}<0$ appears around the $\mathrm{x}$-axis $(y=z=0)$ in $x>X_{\mathrm{C}}$, since the accumulated $B_{\mathrm{Z}}$ field lines are significantly wound (or bent) in the z-direction. Hence, the $B_{\mathrm{y}}$ field always changes its sign at $x=X_{\mathrm{C}}$, since the reconnected field lines in $x<X_{\mathrm{C}}$ have $B_{\mathrm{y}}>0$.

The plasmoid dynamics in the presence of initial sheared fields may be applied to acutual satellite observations in the geomagnetic tail. Here, note again that $B_{\mathrm{x}}, B_{\mathrm{y}}$ and $B_{\mathrm{z}}$ in the present coordinate system correspond to, respectively, $-B_{\mathrm{x}},-B_{\mathrm{z}}$ and $-B_{\mathrm{y}}$ in the conventional GSM coordinates (Fig. 1) (Ugai and Zheng, 2006a, b). When a satellite is located inside the plasmoid, it may observe temporal changes in quantities as the plasmoid propagates in the positive $\mathrm{x}$ direction (Fig. 4). Hence, the magnetic field profiles shown in Figs. 9-12 should be viewed in the negative $\mathrm{x}$-direction. Although the present results are shown only for the initial sheared field $B_{\mathrm{z}}<0$, the underlying physical processes do not depend on its sign. In fact, if $B_{\mathrm{Z}}>0$ is considered, the results are quite the same except that the plasmoid is distorted in the positive $\mathrm{z}$-direction in $y>0$ (Fig. 14b). Hence, because of the $\mathrm{x}$-axis symmetry, the magnetic field profiles in Figs. 9-12 are not changed if the observation position $z$ and the field component $B_{\mathrm{Z}}$ are replaced by $-z$ and $-B_{\mathrm{z}}$, respectively; for instance, for the case of $B_{z 0}=0.1$ and $\alpha=0.1$, the results are the same as those in Fig. 9 if the location $z=-1$ and the curve $B_{\mathrm{z}}$ are replaced by $z=1$ and $-B_{\mathrm{z}}$, respectively. We hence see that the profiles of magnetic field components around the plasmoid center $x=X_{\mathrm{C}}$, shown in Figs. 9 and 12, are in good agreement with the satellite observations shown in Fig. 1. Regarding the magnetic field profiles outside the plasmoid too, we see that those shown in Fig. 13 are in good agreement with the TCR signatures observed in the tail lobe (Slavin, et al., 1993).

The essential aspect of magnetic reconnection is the topological reconfiguration of the magnetic field configuration. 
Contray to the conventional prediction, we demonstrate that inside the plasmoid the reconnected field lines in $x<X_{\mathrm{C}}$ are not helical, since they are not connected topologically to the sheared field lines accumulated in $x>X_{\mathrm{C}}$. Note that the sheared field lines accumulated in $x>X_{\mathrm{C}}$, which were initially embedded in the central current sheet, may easily provide considerable magnitudes of the $B_{\mathrm{y}}$ or the $B_{\mathrm{z}}$ field component according to how the field lines are bent in the z-direction by the plasmoid dynamics (Fig. 14). Also, it may be noted that in addition to the large-scale (principal) plasmoid, magnetic islands are likely to be formed in the diffusion region. In the present model, the island first formed at the origin cannot propagate because of the boundary condition (Figs. 3 and 4), but it can readily propagate if the symmetry boundary condition is not imposed in the diffusion region (Ugai, 1985; Ugai and Zheng, 2006b; Shimizu et al., 2009). Hence, in actual free space, small-scale magnetic islands may subsequently propagate during the proceeding of the fast reconnection.

Unlike the real magnetotail, the present model assumes no initial northward field component in the current sheet. In the presence of such a northward field, if the reconnection starts somewhere in the current sheet, a plasmoid structure with closed or spiral field lines may be formed in the tailward direction. This is the essence of the traditional plasmoid picture (Hones, 1977). In this picture, the x-directional dimension of plasmoid may be determined by that of the outermost closed (spiral) field lines without considering the plasma dynamics directly caused by the reconnection, which is quite different from the present plasmoid formation (Figs. 4 and 5). Hence, in data anlyses for tailward-moving plasmoids one should distinguish between these two effects forming the plasmoid-like objects. On the other hand, there have been observed Earthward-moving plasmoids, where the similar magnetic field signatures like the bipolar signatures of the north-south field component occur (Sergeev et al., 1992; Slavin et al., 2003). In this case, the reconnected field lines have the northward field component, so that the traditional plasmoid picture cannot explain the bipolar signatures of the north-south field component. In fact, Sormakov and Sergeev (2008) recently demonstrated on the basis of satellite observations that the field lines in $x<X_{\mathrm{C}}$ should not be connected topologically to those in $x>X_{\mathrm{C}}$. It may be noted that when the fast reconnection builds up somewhere in the tail current sheet, the fast reconnection jet is simultaneously caused in both the tailward and Earthward directions, so that the resulting tailward-moving and Earthward-moving plasmoids should, in principle, have the same physical properties.

In summary, in the general sheared field geometry, the satellite observations of magnetic fields both inside and outside the plasmoid are well explained by the large-scale (principal) plasmoid generated by the fast reconnection evolution. In addition, on the basis of the spontaneous fast reconnection model, we have successfully applied the 3-D fast reconnection dynamics to well-known signatures of substorms.
Regarding the near-Earth tail, it is widely believed that the so-called substorm current wedge should be formed at the substorm onset (McPherron et al., 1973), and we demonstrated that the current wedge and the generator current circuit drastically evolve from the magnetic loop top (located at $x \sim-10 R_{\mathrm{E}}$ ) when the fast reconnection jet collides with the magnetic loop top (Ugai, 2009a). Also, the satellite observations of Earthward bulk flows were well explained (Kondoh and Ugai, 2008). It is also shown that the different aspects of substorms reflect the different situations where the fast reconnection is triggered (Ugai, 2009b). Recently, it is demonstrated that impulsive magnetic pulsations like the Pi2 pulsations, observed at substorm onsets (Kamide, 1982), can be generated near the loop footpoint (Ugai, 2009c). Hence, it may be said that the spontaneous fast reconnection model is, in principle, responsible for the basic physical mechanism of complicated substorm phenomena.

Acknowledgements. This work was supported by the Grant-in-Aids (21340142) from the Ministry of Education in Japan, Mitsubishi Foundation, RISH of Kyoto University, and Solar-Terrestrial Environment Laboratory of Nagoya University.

Topical Editor I. A. Daglis thanks V. A. Sergeev and another anonymous referee for their help in evaluating this paper.

\section{References}

Hones Jr., E. W.: Substorm processes in the magnetotail: Comments on "On hot tenuous plasma, fireballs, and boundary layers in the Earth's magnetotail” by L.A. Frank et al., J. Geophys. Res., 82, 5633-5640, 1977.

Ieda, A., Machida, S., Mukai, T., Saito, Y., Yamamoto, T., Nishida, A., Terasawa, T., and Kokubun, S.: Statistical analysis of the plasmoid evolution with Geotail observations, J. Geophys. Res., 103, 4453-4465, 1998.

Kamide, Y.: The relationship between field-aligned currents and the auroral electrojets - A review, Space Sci. Rev., 31, 127-243, 1982.

Klimchuk, J. A.: On solving the coronal heating problem, Solar Phys., 234, 41-77, 2006.

Kondoh, K. and Ugai, M.: Numerical studies on three-dimensional earthward fast plasma flows in the near-Earth plasma sheet by the spontaneous fast reconnection model, J. Geophys. Res., 113, A03S07, doi:10.1029/2007JA012707, 2008.

Lui, A. T. Y.: A multiscale model for substorms, Space Sci. Rev., 95, 325-345, 2001.

Lui, A. T. Y.: Potential plasma instabilities for substorm expansion onsets, Space Sci. Rev., 113, 127-206, 2004.

McPherron, R. L., Russel, C. T., and Aurbry, M. P.: Satellite studies of magnetospheric substorms on August 15, 1968. 9. Phenomenological model for substorms, J. Geophys. Res., 78, 31313149, 1973.

Nakamura, R., Baumjohann, W., Asano, Y., Runov, A., Balogh, A., Owen, C. J., Fazakerley, A. N., Fujimoto, M., Klecker, B., and Reme, H.: Dynamics of thin current sheets associated with magnetotail reconnection, J. Geophys. Res., 111, A11206, doi:10.1029/2006JA011706, 2006. 
Petkaki, P. and Freeman, M. P.: Nonlinear dependence of anomalous ion-acoustic resistivity on electron drift velocity, Astrophys. J., 686, 686-693, 2008.

Petschek, H. E.: Magnetic field annihilation, in AAS-NASA Symposium on the Physics of Solar Flares, NASA Spec. Pub., SP-50, 425-439, 1964.

Priest, E. R. and Forbes, T. G.: New models for fast steady state magnetic reconnection, J. Geophys. Res., 91, 5579-5588, 1986.

Sergeev, V. A., Elphic, R. C., Mozer, F. S., Saint-Marc, A., and Sauvaud, J. A.: A two-satellite study of nightside flux transfer events in the plasma sheet, Planet. Space Sci., 40, 1551-1572, 1992.

Sharma, A. S., Nakamura, R., Runov, A., Grigorenko, E. E., Hasegawa, H., Hoshino, M., Louarn, P., Owen, C. J., Petrukovich, A., Sauvaud, J.-A., Semenov, V. S., Sergeev, V. A., Slavin, J. A., Sonnerup, B. U. Ö., Zelenyi, L. M., Fruit, G., Haaland, S., Malova, H., and Snekvik, K.: Transient and localized processes in the magnetotail: a review, Ann. Geophys., 26, 9551006, doi:10.5194/angeo-26-955-2008, 2008.

Shibata, K.: Evidence of magnetic reconnection in solar flares and a unified model of flares, Astrophys. Space Sci., 264, 129-144, 1999.

Shimizu, T., Kondoh, K., Ugai, M., and Shibata, K.: Magnetohydrodynamic study of three-dimensional fast magnetic reconnection for intermittent snake-like downflows in solar flares, Astrophys. J., 707, 420-427, 2009.

Shirataka, N., Fujimoto, M., Hasegawa, H., and TanDokoro, R.: Reproducing the bipolar magnetic signature at the jet leading edge by three-dimensional reconnection with nonzero guide field, J. Geophys. Res., 111, A7201, doi:10.1029/2005JA011521, 2006.

Slavin, J. A., Smith, E. J., Tsurutani, B. T., Sibeck, D. G., Singer, H. J., Baker, D. N., Gosling, J. T., Hones, E. W., and Scarf, F. L.: Substorm associated traveling compression regions in the distant tail - ISEE-3 geotail observations, Geophys. Res. Lett., 11, 657$660,1984$.

Slavin, J. A., Smith, M. F., Mazur, E. L., Baker, D. N., Hones Jr., E. W., Iyemori, T., and Greenstadt, E. W.: ISEE 3 observations of traveling compression regions in the Earth's magnetotail, J. Geophys. Res., 98, 15425-15446, 1993.

Slavin, J. A., Lepping, R. P., Gjerloev, J., Fairfield, D. H., Hesse, M., Owen, C. J., Moldwin, M. B., Nagai, T., Ieda, A., and Mukai, T.: Geotail observations of magnetic flux ropes in the plasma sheet, J. Geophys. Res., 108, 1015, doi:10.1029/93JA01467, 2003.

Sormakov, D. A. and Sergeev, V.: Topology of magnetic flux ropes in the magnetospheric plasma sheet as measured by the Geotail satellite, Cosmic Res., 46, 387-391, 2008.

Ugai, M.: Self-consistent development of fast magnetic reconnection with anomalous plasma resistivity, Plasma Phys. Controlled Fusion, 26, 1549-1563, 1984.

Ugai, M.: Temporal evolution and propagation of a plasmoid associated with asymmetric fast magnetic reconnection, J. Geophys. Res., 90, 9576-9582, 1985.
Ugai, M.: Global dynamics and rapid collapse of an isolated current-sheet system enclosed by free boundaries, Phys. Fluids, 29, 3659-3667, 1986.

Ugai, M.: Computer studies on development of the fast reconnection mechanism for different resistivity models, Phys. Fluids B 4, 2953-2963, 1992.

Ugai, M.: Computer studies on plasmoid dynamics associated with the spontaneous fast reconnection mechanism, Phys. Plasmas, 2, 3320-3328, 1995.

Ugai, M.: Computer studies on the spontaneous fast reconnection model as a nonlinear instability, Phys. Plasmas, 6, 1522-1531, 1999.

Ugai, M.: The evolution of fast reconnection in a threedimensional current sheet system, Phys. Plasmas, 15, 082306 , doi:10.1063/1.2969737, 2008a.

Ugai, M.: Conditions for substorm onset by the fast reconnection mechanism, Ann. Geophys., 26, 3875-3883, doi:10.5194/angeo26-3875-2008, 2008b.

Ugai, M.: Evolution of the current wedge and the generator current circuit by fast reconnection, Phys. Plasmas, 16, 012901, doi:10.1063/1.3055598, 2009a.

Ugai, M.: Fast reconnection evolution in an arcade-like magnetic loop structure, Phys. Plasmas, 16, 062312, doi:10.1063/1.3158949, 2009b.

Ugai, M.: Impulsive magnetic pulsations and electrojets in the loop footpoint driven by the fast reconnection mechanism, Phys. Plasmas, 16, 112902, doi:10.1063/1.3267869, 2009c.

Ugai, M.: Three-dimensional evolution of the fast reconnection mechanism in a sheared current sheet, Phys. Plasmas, 17, 032313, doi:10.1063/1.3369886, 2010a.

Ugai, M.: Three-dimensional evolution of the fast reconnection mechanism in a force-free current sheet, Phys. Plasmas, 17, 06291, doi:10.1063/1.3431097, 2010b.

Ugai, M. and Tsuda, T.: Magnetic field-line reconnexion by localized enhancement of resistivity. Part 1 . Evolution in a compressible MHD fluid, J. Plasma Phys., 17, 337-356, 1977.

Ugai, M. and Zheng, L.: Conditions for the fast reconnection mechanism in three dimensions, Phys. Plasmas, 12, 092312, doi:10.1063/1.2047327, 2005.

Ugai, M. and Zheng, L.: Modeling of traveling compression regions in the Earth's magnetotail by the spontaneous fast reconnection model, Phys. Plasmas, 3, 032901, doi:10.1063/1.2168408, 2006 a.

Ugai, M. and Zheng, L.: Parametric studies on traveling compression regions observed in the Earth's magnetotail, Phys. Plasmas, 13, 062906, doi:10.1063/1.2212827, 2006b.

Uzdensky, D. A.: Petschek-like reconnection with current-driven anomalous resistivity and its application to solar flares, Astrophys. J., 587, 450-457, 2003.

Vasyliunas, V. M.: Theoretical models of magnetic field line merging, Rev. Geophys. Space Phys., 13, 303-336, 1975. 